\title{
Optimierte Therapie in Sicht
}

\section{Differenzierung mithilfe der Quantitativ Sensorischen Testung}

\begin{abstract}
Die Unterscheidung zwischen einem primären und einem sekundären Restless-Legs-Syndrom ist mit der "Quantitativ Sensorischen Testung" (QST) nach DFNS-Standard möglich, wie ein Göttinger Team um Dr. Cornelius Bachmann in Kooperation mit Dr. Roman Rolke, Universität Mainz, in der Fachzeitschrift "Brain" zeigen konnte. Den Forschern gelang es, charakteristische Schmerzprofile für beide Formen zu beschreiben - damit sind Rückschlüsse möglich, welche Therapie für welche Patienten am ehesten geeignet ist.
\end{abstract}

Dund 6-12\% der Bevölkerung westNicher Industrienationen leiden am primären Restless-Legs-Syndrom (RLS). Die Diagnosefindung ist mit einem einzelnen Verfahren nicht möglich. Erschwerend kommt hinzu, dass es neben dem primären RLS auch eine sekundäre Form gibt, die mit einer Erkrankung feiner Nervenfasern, der Small-Fiber-Neuropathie, assoziiert ist.

\section{Die QST als Basis}

Die Forscher konnten nun zeigen, dass eine Unterscheidung mithilfe der QST nach Protokoll des Deutschen Forschungsverbundes Neuropathischer Schmerz (DFNS) möglich ist. Die QSTBatterie des DFNS erfasst mit sieben Tests insgesamt 13 Parameter betreffend die Wahrnehmungs- und Schmerzschwellen für thermische und diverse mechanische Reize (ASUP 1/2008, S. 48ff) [2]. Damit lässt sich das somatosensorische Schmerzprofil der einzelnen Patienten bestimmen.

Das Ergebnis der Studie bei 21 Patienten mit primärem und 13 Patienten mit sekundärem RLS assoziiert mit Small-

\section{Auszeichnung - Förderpreis für Schmerzforschung}

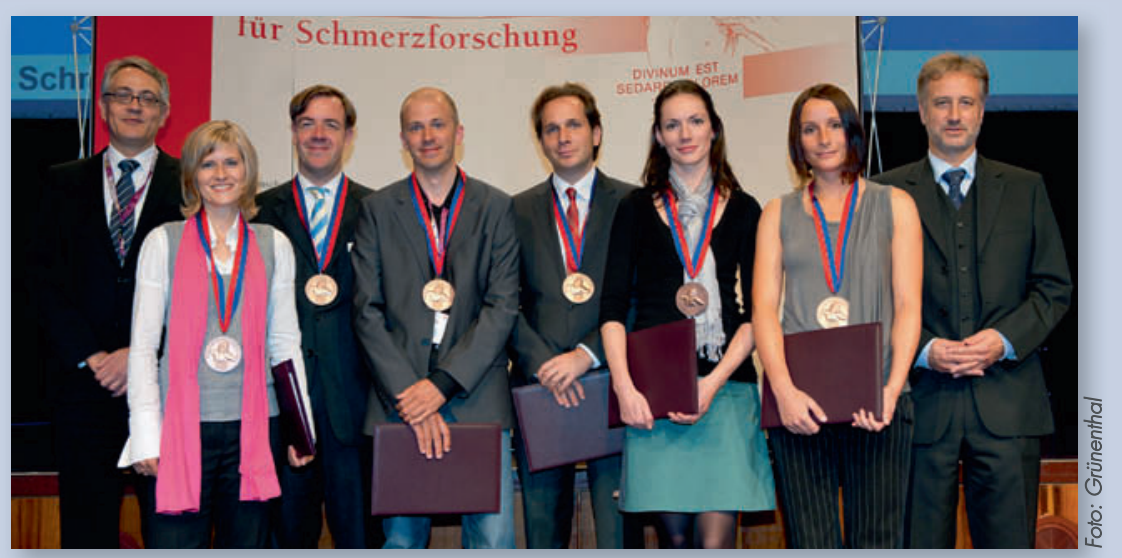

Die Arbeit von Dr. Cornelius Bachmann (3. v. I.) und Dr. Roman Rolke wurde mit dem zweiten Förderpreis für Schmerzforschung 2010 in der Kategorie „Klinische Forschung” prämiert. Der Wissenschaftspreis wird jährlich von der Deutschen Gesellschaft zum Studium des Schmerzes e.V. (DGSS) verliehen und von der Grünenthal GmbH, Aachen, gestiftet. Ganz links: Kai Martens, Geschäftsleiter Deutschland der Grünenthal GmbH; ganz rechts: Prof. Dr. Rolf-Detlef Treede, Mannheim, Präsident der DGSS.

Fiber-Neuropathie im Vergleich mit 20 gesunden Probanden [1]: Zwar waren alle Patienten besonders empfindlich auf spitze Nadelreize, aber Patienten mit primärem RLS nahmen Druck und Vibration stärker wahr. Patienten mit sekundärem RLS mit Erkrankung feiner Nervenfasern zeigten hingegen eine erhöhte Wahrnehmungsschwelle für Kälte und Wärme.

\section{Passgenaue Therapie im Blick}

"Mit der QST können wir nicht nur zwischen den beiden Formen des RestlessLegs-Syndroms unterscheiden - wir sind damit auch in der Lage, die Therapie zu optimieren", freven sich Dr. Bachmann und Dr. Rolke. Patienten, die ein sekundäres RLS assoziiert mit einer Erkrankung feiner Nervenfasern haben, helfen eher Medikamente gegen neuropathischen Schmerz. Patienten mit einem primären RLS profitieren eher von dopaminergen Wirkstoffen.

Literatur

Cornelius G. Bachmann, Roman Rolke, Uta Scheidt, Christine Stadelmann, Martin Sommer, Goran Pavlakovic, Svenja Happe, Rolf-Detlef Treede, Walter Paulus (2010) Thermal hypoaesthesid differentiates secondary restless legs syndrome associated with small fibre neuropathy from primary restless legs syndrome. Brain 133(3): 762-770

2 Rolke R, Baron R, Maier C, Tölle TR, Treede RD, Beyer A, Binder A, Birbaumer N, Birklein F, Bötefür IC, Braune S, Flor H, Huge V, Klug R, Landwehrmeyer GB, Magerl W, Maihöfner C, Rolko C, Schaub C, Scherens A, Sprenger T, Valet M, Wasserka B. (2006) Quantitative Sensory Testing in the German Research Network on Neuropathic Pain (DFNS): Standardized Protocol and Reference Values. Pain 123(3):231-243

Redaktion: Vedrana Romanovic, Pressestelle des DFNS (www. neuropathischer-schmerz.de) 